The following paper posted here is not the official IEEE published version. The final published version of this paper can be found in ICON 2007: IEEE International Conference on Networks (15th : 2007 : Adelaide, Australia):pp.1-6

Copyright (c) 2007 IEEE.

Personal use of this material is permitted. However, permission to reprint/republish this material for advertising or promotional purposes or for creating new collective works for resale or redistribution to servers or lists, or to reuse any copyrighted component of this work in other works must be obtained from the IEEE. 


\title{
Node Localisation in Wireless Ad Hoc Networks
}

\author{
Jon Arnold ${ }^{* \dagger}$, Nigel Bean ${ }^{\dagger}$, Miro Kraetzl ${ }^{*}$, Matthew Roughan ${ }^{\dagger}$ \\ *Defence Science and Technology Organisation \\ PO Box 1500, Edinburgh, S.A. 5111, Australia \\ Email: \{jon.arnold, miro.kraetzl\}@dsto.defence.gov.au \\ ${ }^{\dagger}$ School of Mathematical Sciences \\ University of Adelaide, SA 5005, Australia \\ Email:\{nigel.bean,matthew.roughan\}@ adelaide.edu.au
}

\begin{abstract}
Wireless ad hoc networks often require a method for estimating their nodes' locations. Typically this is achieved by the use of pair-wise measurements between nodes and their neighbours, where a number of nodes already accurately know their location and the remaining nodes must calculate theirs using these known locations. Typically, a minimum mean square estimate (MMSE), or a maximum likelihood estimate (MLE) is used to generate the unknown node locations, making use of range estimates derived from measurements between the nodes. In this paper we investigate the efficacy of using radio frequency, received signal strength (RSS) measurements for the accurate location of the transmitting nodes over long ranges. We show with signal strength measurements from three or more wireless probes in noisy propagation conditions, that by using a weighted MMSE approach we can obtain significant improvements in the variance of the location estimate over both the standard MMSE and MLE approaches.
\end{abstract}

Keywords: Wireless Networks, Location, MMSE, MLE, RSS.

\section{INTRODUCTION}

The localisation of nodes within Wireless Ad Hoc Networks (WAHNs) continues to be the focus of many research efforts. Accurate node localisation is often critical in WAHNs, particularly sensor networks, where this information is crucial to both the observed data and the mechanisms by which the sensing nodes deploy and optimise their collection and communication architectures. Often the nodes deployed in these networks are required to be simple devices that use the minimum of complexity and power to perform their designated functions. The drivers for this are many, with cost, size and operational life under battery power, being the most important, particularly when hundreds of nodes are being deployed to sense a large area. For this reason it is common for the majority of the network nodes to calculate their location based on range estimates derived from measurements to a select few nodes, that accurately know their location from either prior defined information or from additional capability, such as a Global Positioning System (GPS) receiver.

The localisation problem we consider here, is to investigate the efficacy of using radio frequency, received signal strength (RSS) measurements for the accurate location of transmitting nodes over long ranges. Previous research has highlighted that the localisation accuracy reduces with increasing range [1], for the RSS case. For our scenario, we are concerned with the vulnerability of WAHNs to topology reconstruction and characterisation in a military sense. In a previous paper [2], we showed that for an overly simplified propagation model it is possible to approximately reconstruct both the physical and logical topology of a wireless network and characterise individual nodes over time. In this paper, we are interested in optimising the localisation, which is critical for wireless topology reconstruction. The focus is on longer propagation ranges in open terrain, which differs considerably from the often studied problem of locating a transmitting node in an office environment. A more realistic log-normal shadowing propagation model is used, where the propagation exponent that describes the decay in received signal strength proportional to range is set to $n=2$. This is significantly lower than the value used in an office environment, and results in more ideal propagation over longer ranges than experienced within a building. However extending the range worsens the localisation performance due to the log-normal tail in the RSS.

The paper's main contributions are a detailed investigation into the performance of multilateration techniques for node localisation using minimum mean square estimate (MMSE) approaches, and the development of a simple technique to improve the localisation performance by weighting the MMSE. We compare various weighting schemes and show that significant per measurement location bias, that is dependent on the selection of the reference measurement equation, can be introduced if only a simple weighting approach is used. To overcome this limitation, we develop a covariance matrix weighting approach whose performance is independent of the reference equation selected. We compare our technique to the maximum likelihood estimate (MLE) technique developed by Patwari et al. [3] and show that it performs better than the MLE for our purposes.

Section II describes the RF propagation environment. The standard multilateration or MMSE approach is described in Section III. Section IV investigates simple weighting schemes, while Section V uses a mean measurement equation to solve the MMSE. Section VI develops the covariance weighting approach, and this weighted MMSE is compared to the MLE in Section VII. We conclude and propose future work in Section VIII. 


\section{Preliminaries}

Estimating a transmitting node's location from RF signal strength measurements is fraught with much uncertainty. The RF propagation environment can vary markedly, especially with node movement. Fading effects caused by the propagation path or the channel bandwidth can result in large variances in the received signal strength. However, the technique is simple, cheap and the hardware required to estimate the received power already highly integrated in the wireless local area networks (WLAN) receivers used commercially on 802.11 networks and WAHNs.

A log-normal shadowing model was used to simulate the RF propagation loss, with the received signal strength calculated at each node using the distance $d$ between it and the associated nodes. Typically the received power decays proportionally to $d^{-n}$ where $n$ is the path loss exponent. The average received power $P(d)$, at distance $d$ from a transmitting node is typically modelled relative to a small reference distance $d_{o}$, and its received power $P_{o}$. In units of $(\mathrm{dBm})$, we have

$$
P(d)=P_{o}-10 n \log _{10} \frac{d}{d_{o}} .
$$

Rappaport [4] shows that $n$ varies between 2 and 4, and for the free space model $n=2$. It has also been shown that variations in the received power measurements in $\mathrm{dB}$, can be modeled by a Gaussian distribution and that the standard deviation $\sigma$, of the received power can be as low as 4 and as high as 12 [1], [4]. For our simulations, the exact distance from the nodes with known locations, henceforth known as probes, to the unknown node locations was calculated to provide a perfect measurement. Log-normal noise with a mean of zero and a variance of $20 \mathrm{~dB}$, was added to this to simulate the range distance estimates for our open battlefield scenario. This channel model although simplified, provides a good model for our scenario where we assume that there is clear line of sight (CLOS) to our unknown node for the majority of the time and that what variations do occur are from only simple reflections arising from movement or small obstructions. For our simulations we are concerned with only one unknown node as depicted in Figure 1.

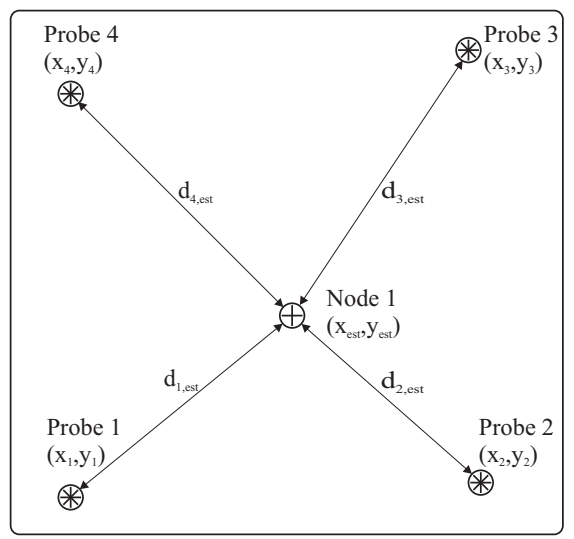

Fig. 1. Probe to node distances.

\section{Node LOCALISATION}

To calculate an estimated node location, the noisy distance measurements are compared to a receiver threshold distance that is calculated according to the sensitivity of a standard 802.11 b receiver plus $6 \mathrm{~dB}$ of loss. The additional loss is included to allow for receiver variations. If this threshold is exceeded for at least three measurement probes, then a MMSE for each node location $\left(x_{e s t}, y_{e s t}\right)$ can be generated. This is often termed multilateration in the WAHN location literature. To solve the MMSE we need $n$ location estimates, where $n \geq 3$. These are calculated by using the probe distance measurements $d_{i, e s t}$, determined from the simulated power measurements via equation (1). Then,

$$
d_{i, e s t}=\sqrt{\left(x_{i}-x_{e s t}\right)^{2}+\left(y_{i}-y_{e s t}\right)^{2}}, i=1, \ldots, n .
$$

Squaring and rearranging these terms yields the following equation for each probe measurement

$$
-x_{i}^{2}-y_{i}^{2}=x_{e s t}^{2}+y_{\text {est }}^{2}-2\left(x_{e s t} x_{i}+y_{\text {est }} y_{i}\right)-d_{i, e s t}^{2} .
$$

For $n$ such probe equations the $x_{e s t}^{2}+y_{\text {est }}^{2}$ can be removed by subtracting the $p_{n}$ probe/reference equation from the set, with the resultant being in the form of $v=\beta A$, where

$$
v=\left[\begin{array}{c}
-x_{1}^{2}-y_{1}^{2}+x_{p_{n}}^{2}+y_{p_{n}}^{2}-d_{n, e s t}^{2}+d_{1, e s t}^{2} \\
-x_{2}^{2}-y_{2}^{2}+x_{p_{n}}^{2}+y_{p_{n}}^{2}-d_{n, \text { est }}^{2}+d_{2, e s t}^{2} \\
\vdots \\
-x_{n-1}^{2}-y_{n-1}^{2}+x_{p_{n}}^{2}+y_{p_{n}}^{2}-d_{n, \text { est }}^{2}+d_{n-1, \text { est }}^{2}
\end{array}\right],
$$

and

$$
\beta=\left[\begin{array}{cc}
2\left(x_{p_{n}}-x_{1}\right) & 2\left(y_{p_{n}}-y_{1}\right) \\
2\left(x_{p_{n}}-x_{2}\right) & 2\left(y_{p_{n}}-y_{2}\right) \\
\vdots & \vdots \\
2\left(x_{p_{n}}-x_{n-1}\right) & 2\left(y_{p_{n}}-y_{n-1}\right)
\end{array}\right]
$$

are known and the unknown node's location estimate is

$$
A=\left[\begin{array}{l}
x_{\text {est }} \\
y_{\text {est }}
\end{array}\right] \text {. }
$$

A is solved using the Moore-Penrose generalised matrix inverse solution for the MMSE [5], [6]

$$
A=\left(\beta^{T} \beta\right)^{-1} \beta^{T} v .
$$

Figure 2 plots the MMSE location and its one-standard deviation uncertainty ellipse $(1-\sigma)$ for a single unknown node calculated using 7000 simulated range estimates from four probes, with the highlighted probes acting as the reference. The one-standard deviation uncertainty ellipse is used as a coarse 2-D confidence interval as in [7]. For a detailed description of this type of uncertainty representation refer to [8].

To provide a measure of the estimated location's variance, we define $\hat{z}=\left(x_{e s t}, y_{\text {est }}\right)$, and $\sigma_{\hat{z}}^{2}$ to be the trace of the location's covariance matrix $\operatorname{cov}(\hat{z})$,

$$
\sigma_{\hat{z}}^{2}=\operatorname{tr}\{\operatorname{cov}(\hat{z})\}
$$


As such, $\sigma_{\hat{z}}$ for the MMSE above is approximately 100 metres, which clearly shows how poor the location estimate can be and that for long range measurements using the standard MMSE is of little use. Further, it appears that whether the probes are placed either approximately symmetric to the node or asymmetrically, that the mean estimated location is largely unbiased.

The log-normal model for the range estimates means that their standard deviation will be proportional to the actual distance separating the probes and nodes. As such the variance for the MMSEs will worsen with increasing distance as shown in the second part of Figure 2. It can also be seen that the uncertainty ellipses for the two MMSEs in each plot are slightly different. This is a result of the fact that with the same data, different reference probes lead to different estimates. Only data with valid (ie above the threshold) readings from the reference probe can be analysed and so there is a degree of biased selection involved for each reference probe. Consequently, if a more distant probe is chosen as the reference probe, less estimates will be generated as less valid measurements are made by that probe. There will also be an associated bias in the number of poor to good distance estimates, as many poor results will drop below the threshold and thus effectively improve the averaged result.

\section{Node Localisation USING A Weighted MMSE}

With the log-normal model resulting in the variance of the range estimates increasing proportionally to the separation distance, it would appear obvious that weighting our measurement matrix inversely proportional to range should improve our MMSE. Accordingly, the weighting matrix $w$,

$$
w=\left[\begin{array}{cccc}
d_{1, e s t}^{n_{w}} & 0 & \cdots & 0 \\
0 & d_{2, \text { est }}^{n_{w}} & \cdots & 0 \\
\vdots & \vdots & \vdots & \vdots \\
0 & 0 & \cdots & d_{n-1, \text { est }}^{n_{w}}
\end{array}\right]
$$

was incorporated into the Moore-Penrose location estimate,

$$
A=\left(\beta^{T} w^{-1} \beta\right)^{-1} \beta^{T} w^{-1} v .
$$

It was anticipated that larger values for the weighting exponent $n_{w}$ in (6), should improve the variance of the location MMSE. To investigate this, the resulting RMS error after 7000 location estimates was plotted for increasing values of $n_{w}$, and is shown in Figure 3. The results clearly suggests that we should increase the weighting exponent to at least 4 . Although larger values further reduce the errors for this well behaved system, they can cause arithmetic problems at higher orders, particularly if the probes and the unknown nodes align colinearly.

Figure 4 shows the mean estimated locations when the MMSE is weighted by $1 / d^{4}$ for the same simulations as in Figure 2. Both plots clearly show that weighting has reduced the variance of the location estimates, but also introduced a significant reference probe dependent bias, in the estimated mean location. For the upper plot, where the probes are
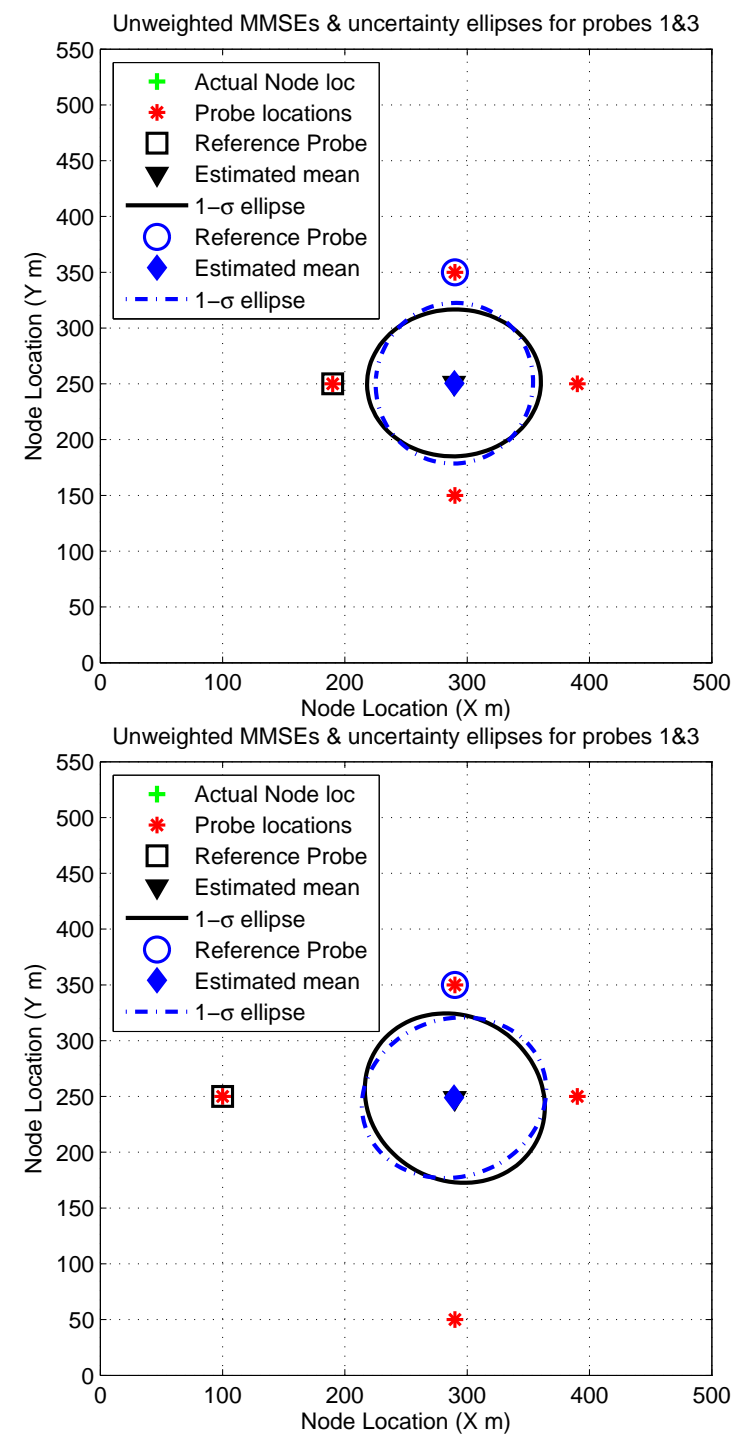

Fig. 2. Unweighted MMSE node locations and their one-standard deviation uncertainty ellipses for approximately symmetric and asymmetric probe placement.

relatively close and symmetric to the unknown node, the bias effects are very similar and clearly orientated relative to the reference probe. For the lower plot, the reference probe dependency is not nearly as obvious. This effect is again a result of the thresholding, which limits the set of possible measurements.

This again highlights, that depending on the relative location of the probes to the unknown node's location, there will be an optimum reference probe choice for the localisation, especially if weighted in this fashion. The problem is that we can't easily tell which probe will produce the best estimate. What is required is a weighting technique that gives robust location estimates independent of the reference probe choice.

\section{MMSE USING A MEAN REFERENCE EQUATION}

In the previous two sections the unknown node's location was calculated by subtracting one probe's distance measure- 


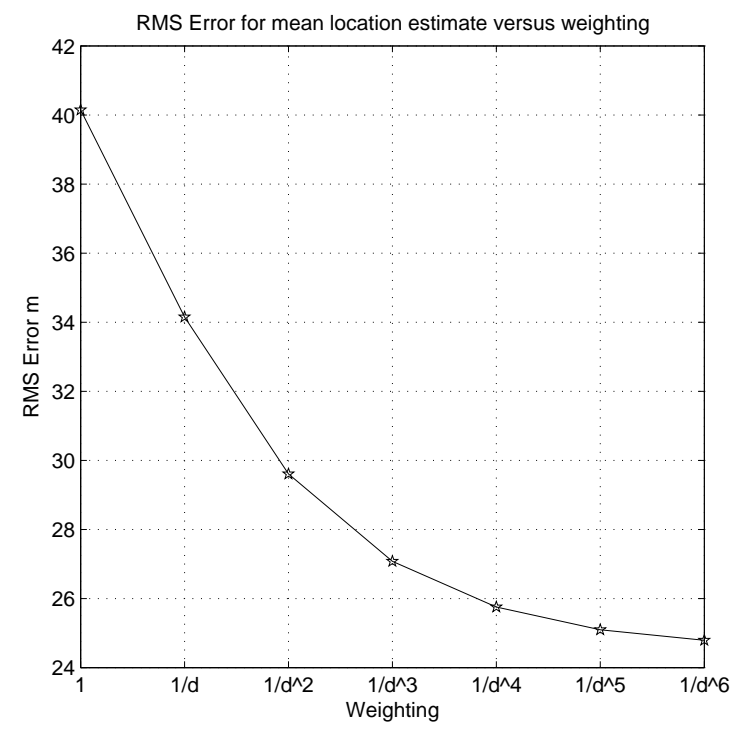

Fig. 3. Plot of RMS error for mean location estimate versus distance weighting exponent.

ment equation (the "reference probe") from the set of probe equations, so that a MMSE for the location could be generated. Instead of subtracting a single probe equation a similar result can be achieved by subtracting the mean of the set of probe measurement equations, thus removing the dependence on a particular probe. The down side of this approach is that probes with very poor estimates will corrupt the average and thus the location estimate. Conversely there may also be an improvement if the variances of the individual probe measurements are all similar. To achieve this, equation (3) is rearranged as below,

$$
d_{i, e s t}^{2}-x_{i}^{2}-y_{i}^{2}=x_{e s t}^{2}+y_{e s t}^{2}-2\left(x_{e s t} x_{i}+y_{e s t} y_{i}\right) .
$$

Define,

$$
\begin{aligned}
& \bar{X}=\frac{1}{N} \sum_{i=1}^{N} x_{i}, \quad \bar{Y}=\frac{1}{N} \sum_{i=1}^{N} y_{i}, \\
& \bar{V}=\frac{1}{N} \sum_{i=1}^{N}\left(d_{i, e s t}^{2}-x_{i}^{2}-y_{i}^{2}\right),
\end{aligned}
$$

Summing (8) over all $i$ and dividing by $\mathrm{N}$ gives,

$$
\bar{V}=x_{e s t}^{2}+y_{\text {est }}^{2}-2 x_{e s t} \bar{X}-2 y_{e s t} \bar{Y} .
$$

Subtracting (8) from (10) for each $i$ we obtain the equations

$$
v_{i}=2\left(x_{i}-\bar{X}\right) x_{e s t}+2\left(y_{i}-\bar{Y}\right) y_{e s t},
$$

where $v_{i}=\bar{V}+x_{i}^{2}+y_{i}^{2}-d_{i, e s t}^{2}$. We can rewrite equation (11) as

$$
\mathbf{v}=\beta A,
$$

where $\beta$ is the known locations as before, just redefined in terms of the means. $\beta=2[\mathbf{x}-\mathbf{1} \bar{X}, \mathbf{y}-\mathbf{1} \bar{Y}]$, where the column vectors $\mathbf{x}=\left(x_{1}, \ldots, x_{N}\right)^{T}, \mathbf{y}=\left(y_{1}, \ldots, y_{N}\right)^{T}$, and $\mathbf{1}=$ $(1,1, \ldots, 1)^{T}$. $A$ is the unknown node's location as before
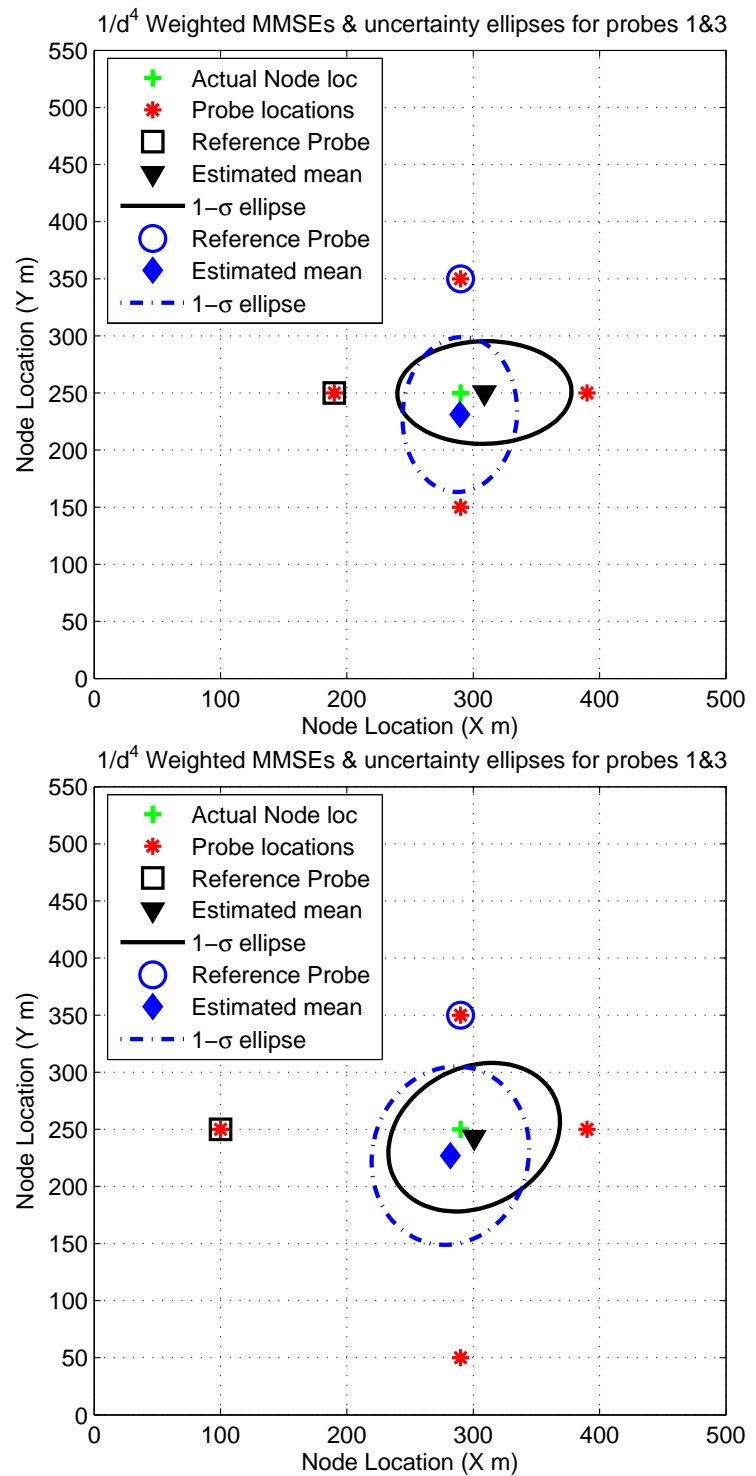

Fig. 4. Plots of mean node location estimates and their one-standard deviation uncertainty ellipses for the selected reference probes when the MMSE is calculated using $1 / d^{4}$ weighting.

and once again is solved using the same weighted MMSE as in equation (7).

Comparing the second plot in Figure 2 with the location estimate generated using the mean reference equation in Figure 5 we see that the mean location estimate is approximately the same with a small improvement in it's variance. However, we have almost completely removed the bias introduced with weighting alone as seen in Figure 4. The estimate's variance however, is still too large and even subtracting a weighted mean equation had no significant effect. Another approach that removes probe dependence and also reduces the estimate's variance is required.

\section{Covariance Weighted MMSE}

Motivated by the fact that weighting a normal distribution via its inverse covariance matrix provides the maximum like- 


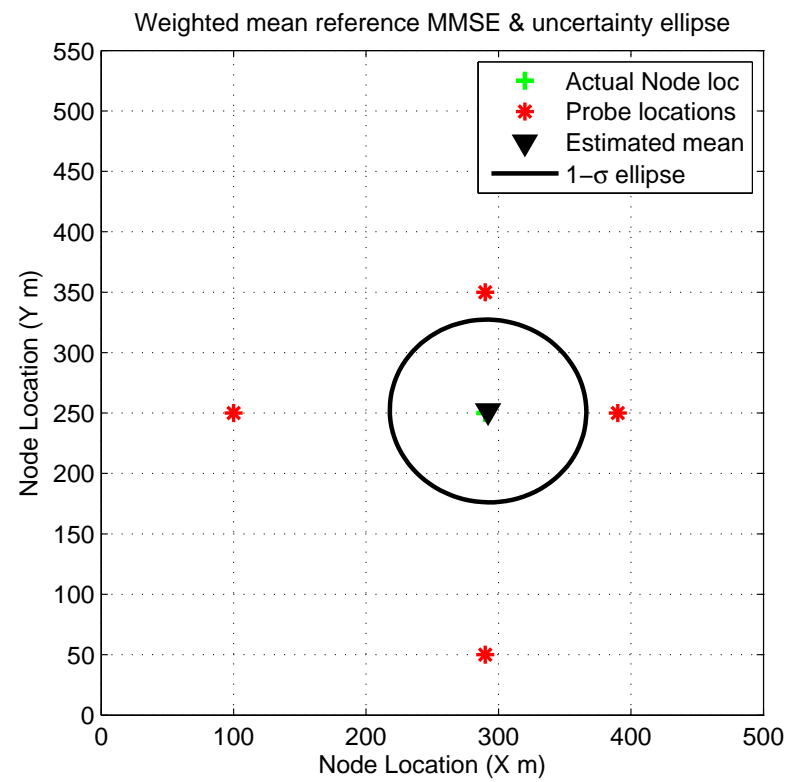

Fig. 5. Weighted mean reference equation MMSE node location and its one-standard deviation uncertainty ellipse.

lihood estimate for the least squares solution, we modify our weighting matrix to include all the covariance terms for the log-normal shadowing case.

For brevity, let $d$ be the distance from the probes to the unknown node. The covariance between the elements of $v$, given that $d_{i}, d_{j}$ and $d_{n}$ are independent, where $\mathrm{n}$ denotes the reference probe, is

$$
\begin{aligned}
\operatorname{cov}\left(v_{i}, v_{j}\right)= & \operatorname{cov}\left(-d_{n}^{2}+d_{i}^{2},-d_{n}^{2}+d_{j}^{2}\right), \\
= & \operatorname{cov}\left(-d_{n}^{2},-d_{n}^{2}\right)+\operatorname{cov}\left(-d_{n}^{2}, d_{j}^{2}\right) \\
& +\operatorname{cov}\left(d_{i}^{2},-d_{n}^{2}\right)+\operatorname{cov}\left(d_{i}^{2}, d_{j}^{2}\right), \\
= & \operatorname{cov}\left(-d_{n}^{2}, d_{n}^{2}\right)+I\{i=j\} \operatorname{cov}\left(d_{i}^{2}, d_{j}^{2}\right), \\
= & \operatorname{var}\left(d_{n}^{2}\right)+I\{i=j\} \operatorname{var}\left(d_{i}^{2}\right) .
\end{aligned}
$$

Consequently the variance for our location estimate for lognormal shadowing is,

$$
\operatorname{cov}\left(v_{i}, v_{j}\right)= \begin{cases}\operatorname{var}\left(d_{n}^{2}\right)+\operatorname{var}\left(d_{i}^{2}\right), & i=j \\ \operatorname{var}\left(d_{n}^{2}\right), & i \neq j\end{cases}
$$

Using the second and fourth raw moments of the log-normal distribution, $\mu_{2}^{\prime}=e^{2 \sigma^{2}}$ and $\mu_{4}^{\prime}=e^{8 \sigma^{2}}$ we can estimate these variances as,

$$
\begin{aligned}
\operatorname{var}\left(d_{i}^{2}\right) & =E\left[\left(d_{i}^{2}\right)^{2}\right]-\left(E\left[d_{i}^{2}\right]\right)^{2} \\
& =E\left[d_{i}^{4}\right]-\left(E\left[d_{i}^{2}\right]\right)^{2} \\
& =d_{i}^{4} e^{8\left(\frac{\sigma \ln 10}{10 n}\right)^{2}}-d_{i}^{4} e^{4\left(\frac{\sigma \ln 10}{10 n}\right)^{2}}, \\
& =d_{i}^{4} C .
\end{aligned}
$$

Our weighting matrix is then the covariance matrix as in (14), which can be written as

$$
w=C\left[\begin{array}{cccc}
d_{n}^{4}+d_{1}^{4} & d_{n}^{4} & \cdots & d_{n}^{4} \\
d_{n}^{4} & d_{n}^{4}+d_{2}^{4} & \cdots & d_{n}^{4} \\
\vdots & \vdots & \vdots & \vdots \\
d_{n}^{4} & d_{n}^{4} & \cdots & d_{n}^{4}+d_{n-1}^{4}
\end{array}\right] .
$$

Surprisingly applying this weighting as in equation (7) results in exactly the same location estimate independent of which probe is chosen to be the reference, for every data set. Figure 6 shows the estimated locations produced by weighting the MMSE by the covariance matrix. The onestandard deviation uncertainty ellipses have been considerably reduced in comparison with both the unweighted and weighted cases. Also significant is that the bias in the mean estimated location is removed or heavily reduced. The results for the first plot show that for the covariance weighted MMSE, $\sigma_{\hat{z}}$ has been reduced to $53.32 \mathrm{~m}$ as compared to those for the $1 / d^{4}$ weighted MMSEs of $82.28 \mathrm{~m}, 82.97 \mathrm{~m}, 81.55 \mathrm{~m}$ and 81.07 $\mathrm{m}$ for probes one to four respectively acting as the reference probe, and $\sigma_{\hat{z}}=96.49 \mathrm{~m}$ for the unweighted case using probe one as the reference probe.
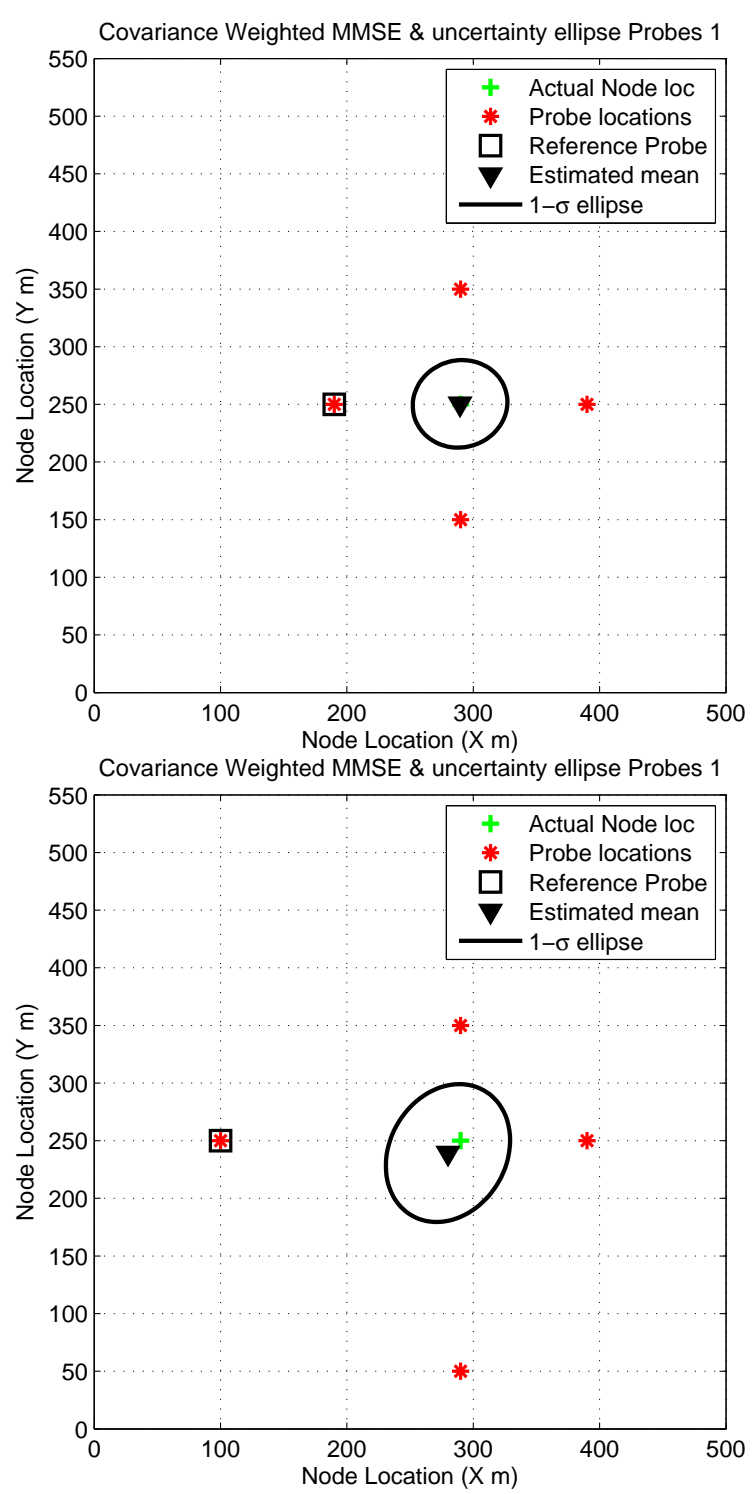

Fig. 6. Plot of mean node location estimates and the one-standard deviation uncertainty ellipses when the MMSE is calculated using the covariance weighting matrix. 
Initial investigations into why the previous weighting matrix provided the same estimate independent of the choice of reference probe were undertaken by expanding equation (7) for an arbitrary weighting matrix with structure

$$
w=\left[\begin{array}{ccc}
W_{4}+W_{1} & W_{4} & W_{4} \\
W_{4} & W_{4}+W_{2} & W_{4} \\
W_{4} & W_{4} & W_{4}+W_{3}
\end{array}\right] .
$$

It appears that the independence of the estimates to reference probe is related to the structure of the covariance matrix. We have yet to prove that the covariance weighting is independent of the choice of reference equation for all situations and leave this for future investigation.

\section{MLE And Covariance Weighted MMSE}

It can be shown that the density of the received power at probe $i$ transmitted from node $j$ (expressed in Watts) $P_{i, j}(W)$ is

$f\left(P_{i, j}(W)\right)=\frac{10 / \log _{e} 10}{\sqrt{2 \pi \sigma_{d B}^{2}}} \frac{1}{P_{i, j}(W)} \exp -\left[\frac{b}{2} \log _{e}\left(\frac{\bar{d}_{i, j}}{\tilde{d}_{i, j}}\right)^{2}\right]$,

where

$$
\begin{aligned}
b & =\left(\frac{10 n}{\sigma_{d B} \log _{e} 10}\right)^{2} \\
\bar{d}_{i, j} & =\text { log-normal mean distance } \\
\tilde{d}_{i, j} & =\text { estimated distance. }
\end{aligned}
$$

Using this density function, Patwari et al. [3] derived the MLE for the unknown node location. This can be simplified to include only those probe-to-node combinations that provide valid range estimates such that the MLE is

$$
\arg \min _{\{z\}} \sum_{i=1}^{n}\left(\log _{e}\left(\frac{\bar{d}_{i, j}^{2}}{\tilde{d}_{i, j}^{2}}\right)\right)^{2}
$$

where $z=\left(x_{\text {est }}, y_{\text {est }}\right)$ represents the potential location of the unknown node. Using (18) we generate a MLE for the unknown node's location for the same scenario. We also use the derivation for the Cramér-Rao bound (CRB) from [1] to compare with the MLE, as shown in Figure 7. Comparing Figures 6 and 7, we see that the $\sigma_{\hat{z}}$ of $53.32 \mathrm{~m}$ for the location estimate provided by the covariance weighted MMSE is considerably better than that for the MLE which is 70.48 $\mathrm{m}$, and approaches the CRB of $51.58 \mathrm{~m}$.

\section{CONCLUSION}

This paper has considered in detail the problem of long range wireless node location estimation using range estimates derived from RSS measurements. We have shown that the estimated location variance can be significantly improved by using the covariance matrix of the measurement equations as a weighting function in the generation of the MMSE. It has also been shown that this approach significantly out performs the MLE and approaches the CRB, for our scenarios.

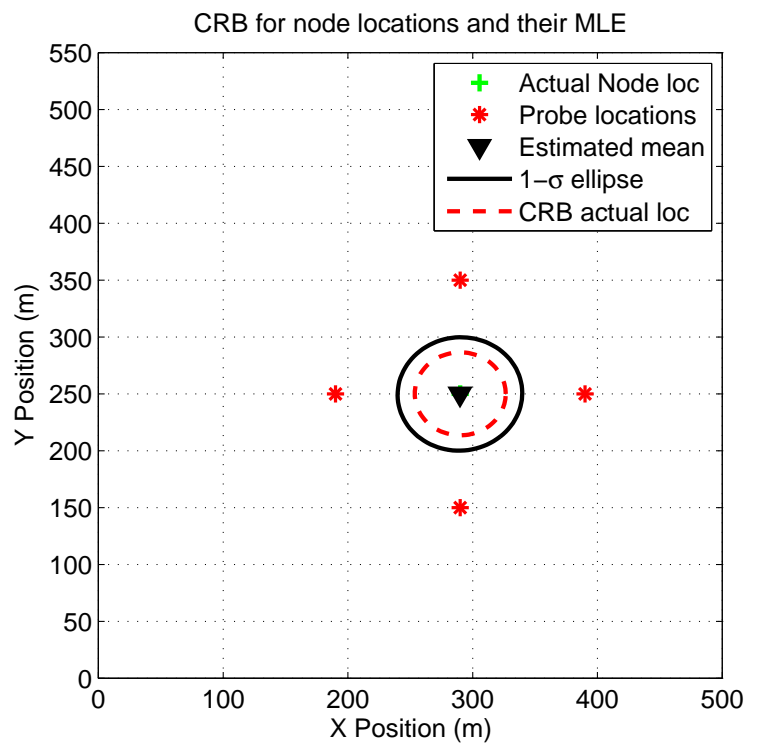

Fig. 7. Plot of mean node location estimates and the one-standard deviation uncertainty ellipses for the MLE.

Future work will further investigate the properties of the covariance weighting approach to ascertain if the independence of the technique to the choice of reference equation holds in general. Similar approaches are currently being investigated for location estimation using time difference of arrival (TDOA) measurements and will be reported in a future paper. We are also exploring the possible benefits of deliberately introducing a distinct form of the variance structure for the estimation process and then exploiting this in tracking and other related problems.

\section{REFERENCES}

[1] N. Patwari, A. O. Hero, M. Perkins, N. Correal, and R. J. O'Dea, "Relative location estimation in wireless sensor networks," IEEE Trans. Signal Processing, vol. 51, pp. 2137-2148, August 2003.

[2] J. Arnold, N. Bean, M. Kraetzl, M. Roughan, and M. Sorell, "Topology reconstruction and characterisation of wireless ad hoc networks," in In Press, The Proceedongs of the International Conference on Communications, 2007.

[3] N. Patwari, R. J. O'Dea, and Y. Wang, "Relative location in wireless networks," in Proc. IEEE VTC, vol. 2, pp. 1149-1153, IEEE, May 2001.

[4] T. S. Rappaport, Wireless Communications Principles \& Practice. New Jersey: Prentice-Hall, 1996.

[5] L. Hogben, Handbook of Linear Algebra. N.W.: Chapman and Hall/CRC, 2007. pp 5.12-5.16.

[6] E. W. Weisstein, "Moore-Penrose matrix inverse." From MathWorld-A Wolfram Web Resource.

[7] N. Patwari, J. Costa, and A. Hero, Locatization In Sensor Networks, ch. Learning signal strength from sensor location and connectivity. Springer-Verlag, 2006.

[8] P. Kontkanen, P. Myllymaki, T. Roos, H. Tirri, K. Valtonen, and H. Wettig, "Topics in probabilistic location estimation in wireless networks," in Proc. 15th IEEE Int. Symposium on Personal, Indoor and Mobile Radio Communications, 2004. 